\title{
Disentangling Genuine from Matter-Induced $C P$ Violation in Neutrino Oscillations
}

\author{
José Bernabéu and Alejandro Segarra \\ Department of Theoretical Physics, University of Valencia and IFIC, University Valencia-CSIC, Burjassot, 46100 Valencia, Spain
}

(Received 21 June 2018; revised manuscript received 4 September 2018; published 21 November 2018)

\begin{abstract}
We prove that, in any flavor transition, neutrino oscillation $C P$-violating asymmetries in matter have two disentangled components: (i) a $C P T$-odd $T$-invariant term, non-vanishing iff there are interactions with matter, and (ii) a $T$-odd $C P T$-invariant term, non-vanishing iff there is genuine $C P$ violation. As function of the baseline, these two terms are distinct $L$-even and $L$-odd observables to separately test (i) matter effects sensitive to the neutrino hierarchy and (ii) genuine $C P$ violation in the neutrino sector. For the golden $\nu_{\mu} \rightarrow \nu_{e}$ channel, the different energy distributions of the two components provide a signature of their separation. At long baselines, they show oscillations in the low and medium energy regions, with zeros at different positions and peculiar behavior around the zeros. We discover a magic energy $E=(0.91 \pm$ $0.01) \mathrm{GeV}$ at $L=1300 \mathrm{~km}$ with vanishing $C P T$-odd component and maximal genuine $C P$ asymmetry proportional to $\sin \delta$, with $\delta$ the weak $C P$ phase. For energies above $1.5 \mathrm{GeV}$, the sign of the $C P$ asymmetry discriminates the neutrino hierarchy.
\end{abstract}

DOI: 10.1103/PhysRevLett.121.211802

The last two decades have seen a revolution in neutrino physics with the discovery of, and precision studies on, flavor oscillations in atmospheric [1], solar [2], reactor [3], and accelerator [4] neutrinos. These phenomena are interpreted in terms of nonvanishing masses and flavor mixing, the unitary Pontecorvo-Maki-Nakagawa-Sakata (PMNS) matrix describing the mismatch between flavor and mass eigenstates. Global fits to all observable quantities provide better and better determination of the two mass differences $\Delta m_{21}^{2}$ and $\left|\Delta m_{31}^{2}\right|$ and the three mixing angles [5-7]. Besides the pending fundamental questions on the DiracMajorana nature of neutrinos and their absolute mass scale, studied by means of other methods, neutrino flavor oscillations have novel challenges for the next-generation experiments like Tokai to Hyper-Kamiokande (T2HK) [8] and the Deep Underground Neutrino Experiment (DUNE) [9]. Above all, once known that the three mixing angles are nonvanishing [10-13], they should answer whether the lepton sector of elementary particles also incorporates $C P$ violation, opening the door to concepts able to explain the matter-antimatter asymmetry of the Universe through leptogenesis [14] at higher energy scales. A second open problem is the ordering of the neutrino mass spectrum, with the so-called normal or inverted hierarchies associated to the positive or negative sign of $\Delta m_{31}^{2}$.

Published by the American Physical Society under the terms of the Creative Commons Attribution 4.0 International license. Further distribution of this work must maintain attribution to the author(s) and the published article's title, journal citation, and DOI. Funded by SCOAP ${ }^{3}$.
Long baseline neutrino oscillation experiments propagate neutrinos from the source, created as muonic flavor, to the detector through the Earth's mantle. The observation of $C P$ violation needs an appearance experiment to a different flavor, and the "suppressed" transition to the electronic flavor is favored. The corresponding $C P$ violation asymmetry, defined in terms of the transition probabilities for neutrinos and antineutrinos $\mathcal{A}_{\alpha \beta}^{C P} \equiv P\left(\nu_{\alpha} \rightarrow \nu_{\beta}\right)-P\left(\bar{\nu}_{\alpha} \rightarrow \bar{\nu}_{\beta}\right)$, is an odd function of $L / E$, with $L$ the baseline and $E$ the relativistic neutrino energy, if and only if the propagation takes place in vacuum. Independent of particular theoretical frameworks, this observable is a bona fide direct proof of $C P$ violation. However, in actual experiments, the propagation takes place in matter, which is $C P$ asymmetric, and a fake $C P$ violation is originated through the different interaction of electron neutrinos and antineutrinos with the electron density of ordinary matter $[15,16]$. This complication in the quest for a direct evidence of fundamental $C P$ violation is widely recognized, and some observables [17-19] have been tried for its separation. On the other hand, matter-induced terms are welcome in order to obtain information on the neutrino mass hierarchy. Because of this combined effect, the generalized attitude in the scientific community has been to extract the $C P$ phase $\delta$ in the $U_{\text {PMNS }}$ mixing matrix from the global fits: a resulting value different from 0 or $\pi$ is taken as evidence of $C P$ violation. Such a methodology is, however, not guaranteeing that the experiment did actually observe $C P$ violation - any quantity sensitive to $\delta$ would make this job, as happens in bare transition probabilities due to the $C P$ conserving $\cos \delta$ terms.

The present Letter represents the restoration of the idea that a direct evidence of symmetry violation means the 
measurement in a single experiment of an observable odd under the symmetry. The concept exploited here is based on the fact that genuine and matter-induced $C P$ violation have opposite behaviors [20] under the other discrete symmetries of time reversal $T$ and $C P T$ : whereas genuine $C P$ violation is odd under $T$ and even under $C P T$, the matter effect is $T$ even and $C P T$ odd. Although they are well separated in the effective Hamiltonian, in general, they are not in the experimental observables and, in particular, in the $C P$ asymmetry. The ideal way to solve this problem would be the independent measurement of $T$-odd and $C P T$-odd asymmetries, but this route requires sources of electron neutrinos and antineutrinos above the muon mass energies, which is, at present, unavailable for accelerator facilities. As an alternative, our work consists in disentangling these two components, genuine and matter-induced $C P$ violation, in the observable $C P$ asymmetry.

Neutrino oscillations in matter are described through the effective Hamiltonian in the flavor basis [15,21-25]

$H=\frac{1}{2 E}\left\{U\left[\begin{array}{ccc}m_{1}^{2} & 0 & 0 \\ 0 & m_{2}^{2} & 0 \\ 0 & 0 & m_{3}^{2}\end{array}\right] U^{\dagger}+\left[\begin{array}{lll}a & 0 & 0 \\ 0 & 0 & 0 \\ 0 & 0 & 0\end{array}\right]\right\}=\frac{1}{2 E} \tilde{U} \tilde{M}^{2} \tilde{U}^{\dagger}$,

where the first term describes neutrino oscillations in vacuum, and the second one accounts for matter effects. The $a$ parameter is given by $a=2 E V$, with $V$ the interaction potential and $E$ the relativistic neutrino energy. For antineutrinos, $U \rightarrow U^{*}$ and $a \rightarrow-a$. All neutrino masses $\left(\tilde{M}^{2}\right)$ and mixings $(\tilde{U})$ in matter, i.e., eigenvalues and eigenstates of $H$, can be calculated in terms of the parameters in the vacuum Hamiltonian $\left(M^{2}, U\right)$ and $a$. Several analytical approaches have been presented in the literature [26-29] using approximations based on the hierarchical values of the different parameters. For later purposes in this Letter, we have developed [30] a perturbative approach in $\Delta m_{21}^{2}-$ against $\Delta m_{31}^{2}$ - for any value of $a$.

The exact Hamiltonian leads to the flavor oscillation probabilities

$$
\begin{aligned}
P\left(\nu_{\alpha} \rightarrow \nu_{\beta}\right)= & \delta_{\alpha \beta}-4 \sum_{j<i} \operatorname{Re} \tilde{J}_{\alpha \beta}^{i j} \sin ^{2} \tilde{\Delta}_{i j} \\
& -2 \sum_{j<i} \operatorname{Im} \tilde{J}_{\alpha \beta}^{i j} \sin 2 \tilde{\Delta}_{i j},
\end{aligned}
$$

where $\tilde{J}_{\alpha \beta}^{i j} \equiv \tilde{U}_{\alpha i} \tilde{U}_{\alpha j}^{*} \tilde{U}_{\beta i}^{*} \tilde{U}_{\beta j}$ are the rephasing-invariant mixings and $\tilde{\Delta}_{i j} \equiv \frac{\Delta \tilde{m}_{i j}^{2} L}{4 E}$. Notice that both $\tilde{J}_{\alpha \beta}^{i j}$ and $\Delta \tilde{m}_{i j}^{2}$ are energy dependent in matter. Because of the $C P V$, CPTV $a \neq 0$ interaction, neutrinos and antineutrinos acquire different masses and the complex mixings do not satisfy the condition $\tilde{\bar{J}}=\tilde{J}^{*}$, where $\tilde{\bar{J}}$ refers to antineutrinos. The $T$-odd genuine $C P$ violation in matter needs, on the other hand, $\tilde{J} \neq \tilde{J}^{*}, \tilde{\bar{J}} \neq \tilde{\bar{J}}^{*}$. From these results on the different behavior under the discrete $T$ and $C P T$ symmetry transformations, one derives the asymmetry disentanglement theorem by separating the observable $C P$ asymmetry in any flavor transition into $L$-even (CPT-violating) and $L$-odd ( $T$-violating) functions,

$$
\begin{aligned}
& \mathcal{A}_{\alpha \beta}^{C P} \equiv P\left(\nu_{\alpha} \rightarrow \nu_{\beta}\right)-P\left(\bar{\nu}_{\alpha} \rightarrow \bar{\nu}_{\beta}\right)=\mathcal{A}_{\alpha \beta}^{C P T}+\mathcal{A}_{\alpha \beta}^{T}, \\
& \mathcal{A}_{\alpha \beta}^{C P T}=-4 \sum_{j<i}\left[\operatorname{Re} \tilde{J}_{\alpha \beta}^{i j} \sin ^{2} \tilde{\Delta}_{i j}-\operatorname{Re} \tilde{\bar{J}}_{\alpha \beta}^{i j} \sin ^{2} \tilde{\bar{\Delta}}_{i j}\right], \\
& \mathcal{A}_{\alpha \beta}^{T}=-2 \sum_{j<i}\left[\operatorname{Im} \tilde{J}_{\alpha \beta}^{i j} \sin 2 \tilde{\Delta}_{i j}-\operatorname{Im} \tilde{\bar{J}}_{\alpha \beta}^{i j} \sin 2 \tilde{\bar{\Delta}}_{i j}\right],
\end{aligned}
$$

where $\mathcal{A}_{\alpha \beta}^{C P T}$ is $T$ invariant and vanishes when $a=0$, whereas $\mathcal{A}_{\alpha \beta}^{T}$ is $C P T$ invariant and vanishes when the flavor mixing is real, corresponding to the $C P$ phase of the PMNS matrix $\delta=0, \pi$. Equations (2), (3) remain valid for extended models with more mass eigenstates and a rectangular mixing matrix.

For the three-neutrino model, the unique rephasing invariant associated to genuine $C P$ violation $\tilde{\mathcal{J}}$ is related to its value in vacuum $\mathcal{J}=c_{12} c_{13}^{2} c_{23} s_{12} s_{13} s_{23} \sin \delta$ [31] by

$$
\tilde{\mathcal{J}} \equiv \operatorname{Im} \tilde{J}_{e \mu}^{12}=\frac{\Delta m_{21}^{2} \Delta m_{31}^{2} \Delta m_{32}^{2}}{\Delta \tilde{m}_{21}^{2} \Delta \tilde{m}_{31}^{2} \Delta \tilde{m}_{32}^{2}} \mathcal{J}
$$

This connection [32] between vacuum and matter is due to the fact that the quantity characterizing $C P$ violation in the flavor basis [33] remains invariant for any flavordiagonal interaction. The proportionality of $\tilde{\mathcal{J}}$ with $\Delta m_{21}^{2}$ in Eq. (4) explains the absence of genuine $C P$ violation in the vanishing limit of this parameter, even if there are three nondegenerate neutrino masses in matter. This transmutation from masses in vacuum to mixings in matter was already observed in Ref. [34], where the limit $\Delta m_{21}^{2}=0$ in vacuum led to $\tilde{U}_{e 1}=0$. The complexity of $\tilde{J}_{\alpha \beta}^{i j}$ is entirely due to $\sin \delta$.

We illustrate, in Fig. 1, the power and expected behavior of the disentanglement theorem by a separate representation of $\mathcal{A}_{\mu e}^{C P T}$ and $\mathcal{A}_{\mu e}^{T}$ as a function of the matter potential $a$ and the $C P$ phase $\delta$. The chosen $(E, L)$ point gives comparable values of the two components, showing their appropriate parities under these two parameters.

This and other results are produced from a full numerical calculation of the $\nu_{\mu} \rightarrow \nu_{e}$ transition produced by the exact Hamiltonian (1), using the best-fit mixing parameters from Ref. [5] and assuming normal hierarchy, unless otherwise specified. Figure 1 shows that $\mathcal{A}_{\alpha \beta}^{C P T}$ is even in $\sin \delta \forall a$, and vanishes at $a=0 \forall \delta$, whereas $\mathcal{A}_{\alpha \beta}^{T}$ is odd in $\sin \delta \forall a$, as requested by the theorem. 


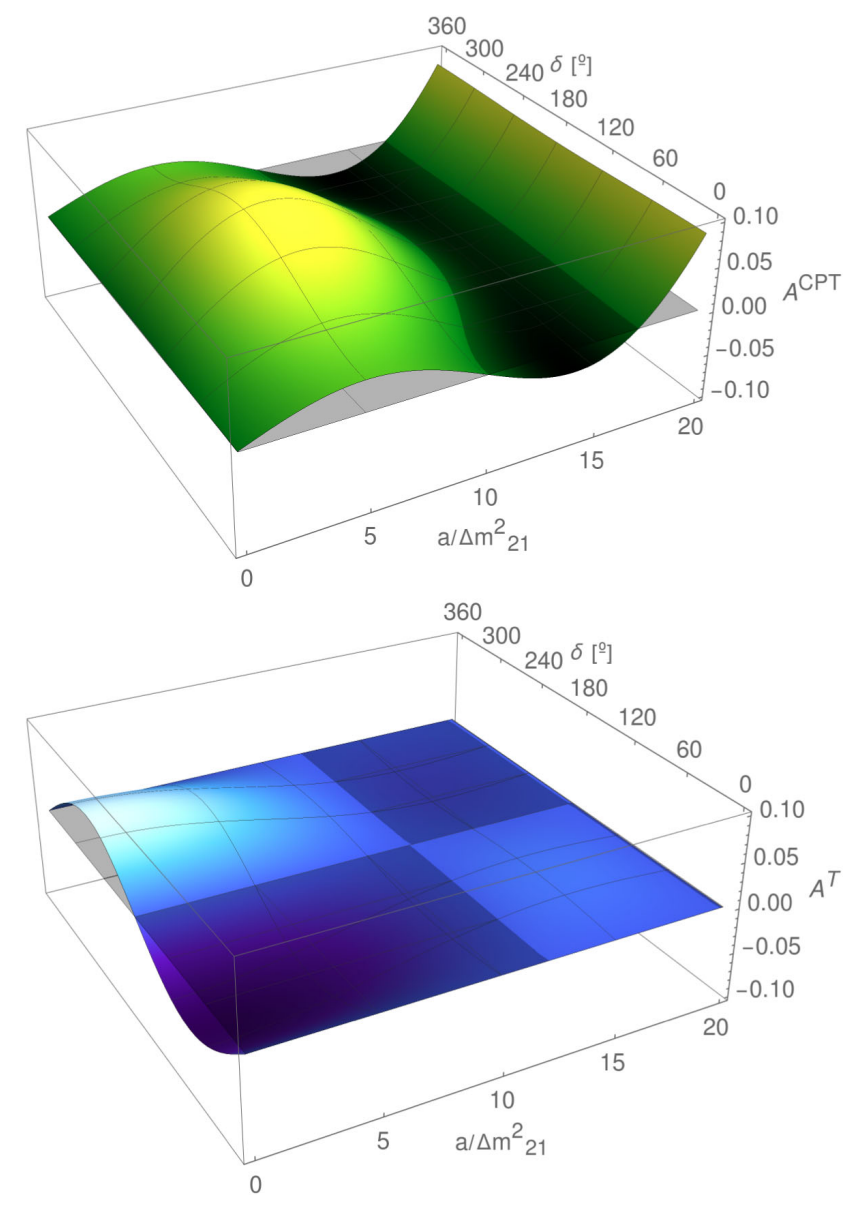

FIG. 1. $\quad C P T$-odd (up) and $T$-odd (down) components of $\mathcal{A}_{\mu e}^{C P}$ at $L=1300 \mathrm{~km}$ and $E=0.75 \mathrm{GeV}$ as a function of $a$ and $\delta$.

For neutrino propagation through the Earth's mantle, valid for terrestrial accelerator neutrinos, the value of the matter potential [35] allows us to write $a \approx 3(E / \mathrm{GeV}) \Delta m_{21}^{2}$. In the energy region between the two Mikheyev-SmirnovWolfenstein resonances, $\Delta m_{21}^{2} \ll a \ll\left|\Delta m_{31}^{2}\right|$, valid for both $\mathrm{T} 2 \mathrm{HK}$ and DUNE neutrino energy spectra, one may approximate the relation in Eq. (4) between $\tilde{\mathcal{J}}$ in matter and $\mathcal{J}$ in vacuum as [30]

$\tilde{\mathcal{J}} \approx \frac{\Delta m_{21}^{2} \Delta m_{31}^{2}}{|a|\left(1-\left|U_{e 3}\right|^{2}\right) \sqrt{\left(\Delta m_{31}^{2}-a\right)^{2}+4 a \Delta m_{31}^{2}\left|U_{e 3}\right|^{2}}} \mathcal{J}$,

where the proportionality factor is energy dependent through $a$.

In the following, we explore whether, at fixed $L$ through the Earth's mantle, the energy distributions of $\mathcal{A}_{\mu e}^{C P T}$ and $\mathcal{A}_{\mu e}^{T}$ present signatures of their separation in $\mathcal{A}_{\mu e}^{C P}$ for the T2HK and DUNE experiments.

Figure 2 gives the predictions for the energy distributions of $\mathcal{A}_{\mu e}^{C P T}$ and $\mathcal{A}_{\mu e}^{T}$ at an intermediate baseline $L=295 \mathrm{~km}$. It is worth noting the lack of oscillating structure in the neutrino energy for both terms of the $C P$ asymmetry. The

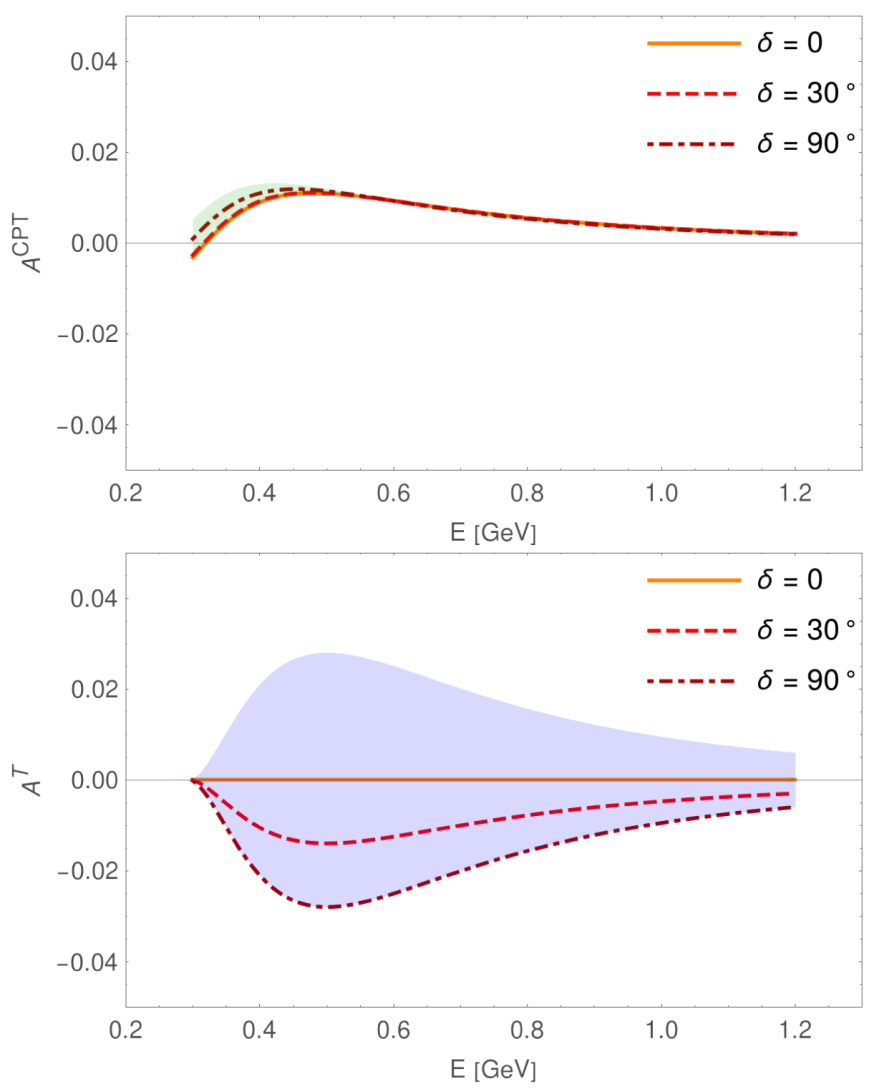

FIG. 2. Energy distribution of the $C P T$-odd (up) and $T$-odd (down) components of the $C P$ asymmetry for the T2HK experiment. The bands correspond to all possible values changing $\delta$ in $(0,2 \pi)$.

magnitude of $\mathcal{A}_{\mu e}^{C P T}$ is small, as expected, and slightly dependent on the $C P$ phase $\delta$ through the genuine $C P$ conserving small contributions of order $\Delta m_{21}^{2}$, more visible at low energies. When these results for a normal hierarchy are recalculated for an inverted hierarchy, the net effect is essentially a change of sign in $\mathcal{A}_{\mu e}^{C P T}$. On the contrary, the magnitude of $\mathcal{A}_{\mu e}^{T}$ is proportional to $\sin \delta$, as predicted by Eq. (4), without any degeneracy when $\delta$ is varied in the entire interval from 0 to $2 \pi$. In addition, in this genuine term of the $C P$ asymmetry the hierarchy in the neutrino mass spectrum plays no role: its sign remains invariant when the sign of the largest mass splitting is changed. We conclude that the sign of $\mathcal{A}_{\mu e}^{C P T}$ fixes the hierarchy, whereas the magnitude and sign of $\mathcal{A}_{\mu e}^{T}$ fixes $\sin \delta$.

The beautiful different behavior of $\mathcal{A}_{\alpha \beta}^{C P T}$ and $\mathcal{A}_{\alpha \beta}^{T}$ for the discrimination of the hierarchy is well understood to leading order in $\Delta m_{21}^{2}$ : zeroth order for $\mathcal{A}_{\alpha \beta}^{C P T}$, independent of $\delta$, and first order for $\mathcal{A}_{\alpha \beta}^{T}$. The mass spectrum in matter changes from neutrinos to antineutrinos as

$\Delta \tilde{m}_{21}^{2} \leftrightarrow \Delta \tilde{\bar{m}}_{21}^{2}, \quad \Delta \tilde{m}_{31}^{2} \leftrightarrow-\Delta \tilde{\bar{m}}_{32}^{2}, \quad \Delta \tilde{m}_{32}^{2} \leftrightarrow-\Delta \tilde{\bar{m}}_{31}^{2}$. 
Under this exchange of neutrinos by antineutrinos, the imaginary part of $\tilde{J}_{\alpha \beta}^{i j}$, as that of $J_{\alpha \beta}^{i j}$, changes sign, whereas the real parts do not. As the $C P$ asymmetries are a difference between neutrino and antineutrino oscillation probabilities, we discover that $\mathcal{A}_{\alpha \beta}^{C P T}$ is changing its sign, whereas the sign of $\mathcal{A}_{\alpha \beta}^{T}$ remains invariant under the change of hierarchy, as seen in our numerical results.

The increase in the baseline from $L=295$ to $L=$ $1300 \mathrm{~km}$ has a very important implication: the appearance of oscillations in the low and medium neutrino energy regions of the two distributions. There is a different pattern for the two components $\mathcal{A}_{\mu e}^{C P T}$ and $\mathcal{A}_{\mu e}^{T}$ of the experimental $C P$ asymmetry, with the zeros at different values and $\mathcal{A}_{\mu e}^{C P T}$ changing its sign around the zeros, whereas $\mathcal{A}_{\mu e}^{T}$ does not. This contrast is very well apparent in the results we show in Fig. 3.

Besides this additional effect of having, in the different energy distributions, a signature to separate the two components, all the other properties discussed above remain the same, independent of the baseline. These are the slight dependence of $\mathcal{A}_{\mu e}^{C P T}$ on $\delta$ due to effects of $\Delta m_{21}^{2}$ at low energies and the hierarchy discrimination with its sign, as well as the proportionality of $\mathcal{A}_{\mu e}^{T}$ with $\sin \delta$ independent of the neutrino hierarchy.
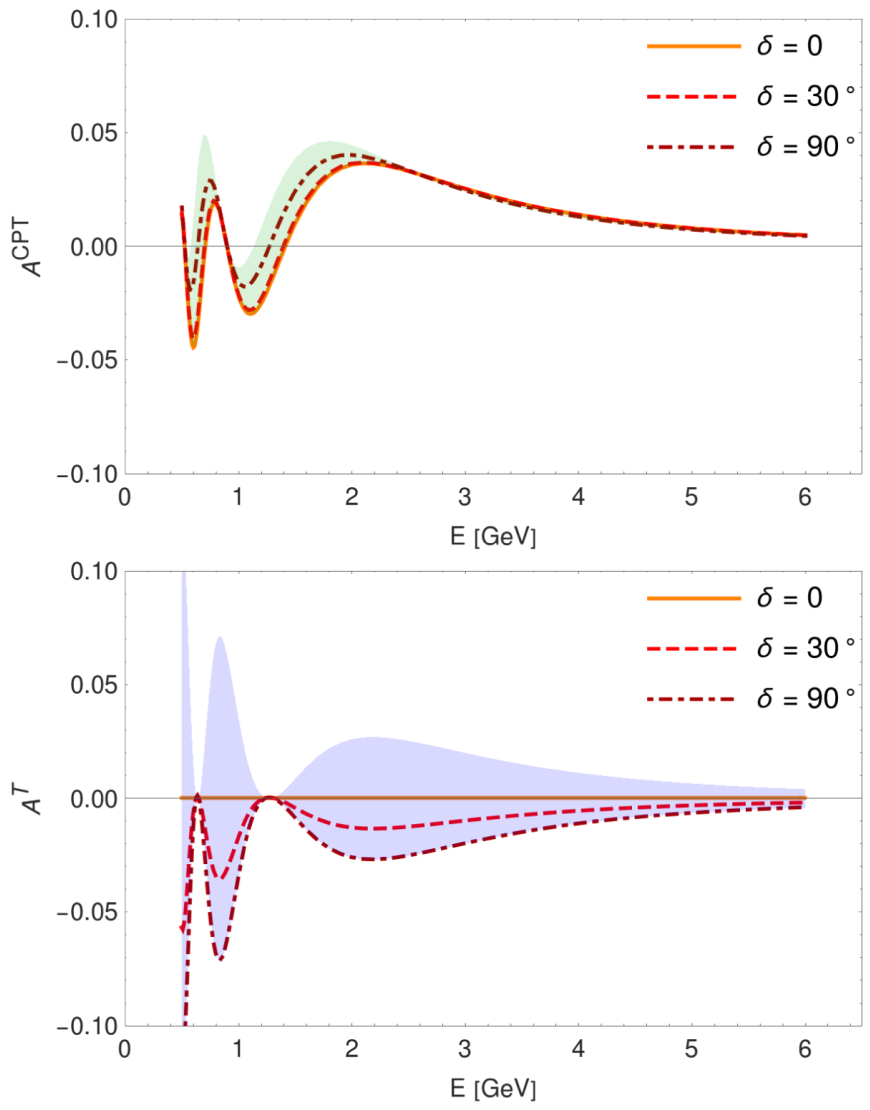

FIG. 3. As Fig. 2 for the DUNE experiment. Notice a factor of 2 in the asymmetry scale with respect to Fig. 2.
In our scan of the different energy distribution behavior at $L=1300 \mathrm{~km}$ of the matter-induced $\mathcal{A}_{\mu e}^{C P T}$ and genuine $\mathcal{A}_{\mu e}^{T}$ components of the $C P$ asymmetry, we have discovered a magic energy interval around $E=0.91 \mathrm{GeV}$ with a zero for $\mathcal{A}_{\mu e}^{C P T}$ and a relative maximum for $\left|\mathcal{A}_{\mu e}^{T}\right|$. We checked that this energy value changes linearly with the vacuum $\left|\Delta m_{31}^{2}\right|$ and is both hierarchy independent and blind to all other fit parameters. This remarkable configuration is well seen in the results presented in Fig. 4, with the two bands for $\mathcal{A}_{\mu e}^{C P T}$ (green area) and $\mathcal{A}_{\mu e}^{T}$ (blue area). The zero in $\mathcal{A}_{\mu e}^{C P T}$ is independent of $\delta$, and $\mathcal{A}_{\mu e}^{C P T}$ changes its sign around this first-order zero, whereas $\mathcal{A}_{\mu e}^{T}$ has a maximal value proportional to $\sin \delta$. This behavior ensures that, for a bin width up to the feasible [36] $0.15 \mathrm{GeV}$, the mean value of $\mathcal{A}_{\mu e}^{C P T}$ is below $10 \%\left|\mathcal{A}_{\mu e}^{T}\right|_{\max }$, whereas $\mathcal{A}_{\mu e}^{T}$ is above $95 \%$ of its peak value.

To summarize, in this Letter, we have provided a conceptual basis for disentangling genuine from matterinduced $C P$ violation in neutrino oscillations. This is achieved by means of a theorem in which the experimental $C P$ asymmetry contains two terms with different behavior under $T$ and $C P T$ symmetries. Whereas the matter-induced component is an even function of the baseline, the genuine $C P$ violating term is odd in $L$, as it is for the case in vacuum. In this separation, $\mathcal{A}_{\alpha \beta}^{C P}=\mathcal{A}_{\alpha \beta}^{C P T}+\mathcal{A}_{\alpha \beta}^{T}$, we have demonstrated that (i) $\mathcal{A}_{\alpha \beta}^{C P T}$ is even in $\sin \delta \forall a$, and vanishes at $a=0 \forall \delta$, whereas $\mathcal{A}_{\alpha \beta}^{T}$ is odd in $\sin \delta \forall a$. (ii) $\mathcal{A}_{\alpha \beta}^{C P T}$, which is slightly dependent on $\delta$ at low energies, changes its sign in going from the normal to the inverted hierarchy, whereas $\mathcal{A}_{\alpha \beta}^{T}$ remains the same.

For flavor-diagonal interactions with matter, the genuine $C P$ violation condition in the flavor basis remains the same for the propagation in matter and in vacuum. This implies a well defined proportionality between the genuine $C P$-odd rephasing-invariant mixings

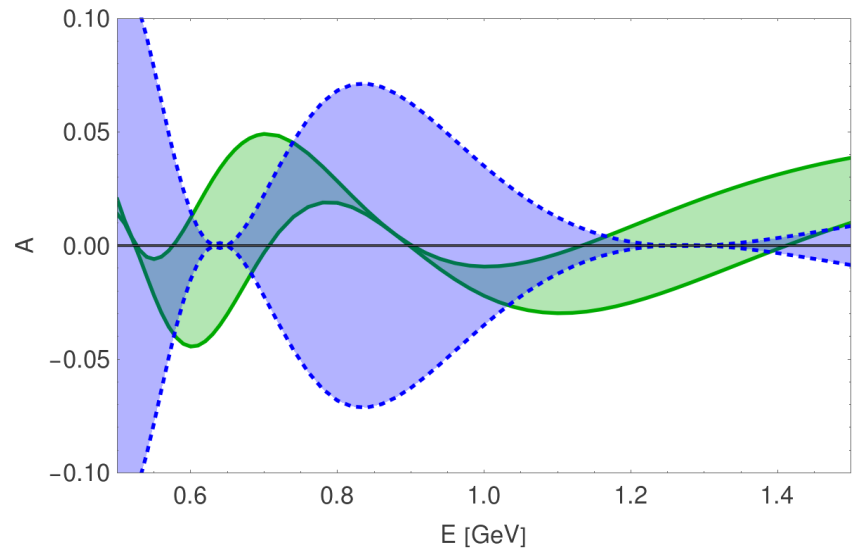

FIG. 4. Zooming Fig. 3 at low E, superposing the $C P T$-odd (green, solid) and $T$-odd (blue, dashed) asymmetries. 
for both cases, the proportionality factor being given by energy-dependent neutrino masses in matter.

The planned experiments for terrestrial neutrinos propagating through the Earth's mantle look for the $C P$ asymmetry in the golden channel $\nu_{\mu} \rightarrow \nu_{e}$ at fixed baselines $L$ and continuum energy spectra $E$ of the neutrino beam. In the search of appropriate signatures for the separation of the two components of the $C P$ asymmetry, we have studied the two energy distributions, presenting peculiar differences at a fixed long baseline: (i) The zeros are at different energies for $\mathcal{A}_{\mu e}^{C P T}$ and $\mathcal{A}_{\mu e}^{T}$, and their behavior around zero is different: $\mathcal{A}_{\mu e}^{C P T}$ changes sign whereas $\mathcal{A}_{\mu e}^{T}$ does not. (ii) In particular, we have discovered a magic configuration around $E=0.91 \mathrm{GeV}$ at $L=1300 \mathrm{~km}$, which presents a zero for $\mathcal{A}_{\mu e}^{C P T}$, independent of $\delta$, and an extremal value for $\mathcal{A}_{\mu e}^{T}$ proportional to $\sin \delta$. This energy value depends linearly on $\left|\Delta m_{31}^{2}\right|$. Calculated for $\left|\Delta m_{31}^{2}\right|=(2.5 \pm 0.03) \times 10^{-3} \mathrm{eV}^{2}$, the magic energy is $E=(0.91 \pm 0.01) \mathrm{GeV}$. At this hierarchy-independent point, a measurement of $\mathcal{A}_{\mu e}^{C P}$ with energy resolution up to $0.15 \mathrm{GeV}$ directly probes genuine $C P$ violation in the lepton sector. (iii) For energies above $1.5 \mathrm{GeV}$, the sign of the $C P$ asymmetry discriminates the neutrino hierarchy.

The authors would like to acknowledge fruitful discussions with Francisco Botella, Anselmo Cervera, and Sergio Palomares. This research has been supported by Spanish Ministerial Department of Research (MINECO) Project No. FPA 2017-84543-P, Generalitat Valenciana Project No. GV PROMETEO 2017-033, and Severo Ochoa Excellence Centre Project No. SEV 2014-0398. A. S. acknowledges the Spanish Ministry of Education (MECD) support through the Grant No. FPU14/04678.

[1] Y. Fukuda et al. (Super-Kamiokande Collaboration), Phys. Rev. Lett. 81, 1562 (1998).

[2] Q. R. Ahmad et al. (SNO Collaboration), Phys. Rev. Lett. 89, 011301 (2002).

[3] T. Araki et al. (KamLAND Collaboration), Phys. Rev. Lett. 94, 081801 (2005).

[4] M. H. Ahn et al. (K2K Collaboration), Phys. Rev. D 74, 072003 (2006).

[5] P. F. de Salas, D. V. Forero, C. A. Ternes, M. Tortola, and F. W. F. Valle, Phys. Lett. B 782, 633 (2018).

[6] I. Esteban, M. C. Gonzalez-Garcia, M. Maltoni, I. MartinezSoler, and T. Schwetz, J. High Energy Phys. 01 (2017) 087.

[7] F. Capozzi, G. L. Fogli, E. Lisi, A. Marrone, D. Montanino, and A. Palazzo, Phys. Rev. D 89, 093018 (2014).
[8] K. Abe et al. (Hyper-Kamiokande Proto Collaboration), ICRR Report No. 701-2016-1 2016, https://lib-extopc.kek .jp/preprints/PDF/2016/1627/1627021.pdf.

[9] R. Acciarri et al. (DUNE Collaboration), Fermilab Report No. FERMILAB-DESIGN-2016-02, arXiv:1512.06148.

[10] K. Abe et al. (T2K Collaboration), Phys. Rev. Lett. 107, 041801 (2011).

[11] Y. Abe et al. (Double Chooz Collaboration), Phys. Rev. Lett. 108, 131801 (2012).

[12] F. An et al. (Daya Bay Collaboration), Phys. Rev. Lett. 108, 171803 (2012).

[13] J. K. Ahn et al. (RENO Collaboration), Phys. Rev. Lett. 108, 191802 (2012).

[14] M. Fukugita and T. Yanagida, Phys. Lett. B 174, 45 (1986).

[15] L. Wolfenstein, Phys. Rev. D 17, 2369 (1978).

[16] S. P. Mikheyev and A. Yu. Smirnov, Sov. J. Nucl. Phys. 42, 913 (1985).

[17] A. Donini, M. B. Gavela, P. Hernandez, and S. Rigolin, Nucl. Phys. B574, 23 (2000).

[18] E. Kh. Akhmedov, M. Maltoni, and A. Yu. Smirnov, J. High Energy Phys. 06 (2008) 072.

[19] T. Ohlsson, H. Zhang, and S. Zhou, Phys. Rev. D 87, 053006 (2013).

[20] J. Bernabeu and M. C. Banuls, Nucl. Phys. B, Proc. Suppl. 87, 315 (2000).

[21] V. Barger, K. Whisnant, S. Pakvasa, and R. J. N. Phillips, Phys. Rev. D 22, 2718 (1980).

[22] T. K. Kuo and J. Pantaleone, Rev. Mod. Phys. 61, 937 (1989).

[23] H. W. Zaglauer and K. H. Schwarzer, Z. Phys. C 40, 273 (1988).

[24] P. Krastev, Nuovo Cimento A 103, 361 (1990).

[25] R. H. Bernstein and S. J. Parke, Phys. Rev. D 44, 2069 (1991).

[26] A. Cervera, A. Donini, M. B. Gavela, J. J. Gomez Cadenas, P. Hernandez, O. Mena, and S. Rigolin, Nucl. Phys. B579, 17 (2000); B593, 731(E) (2001).

[27] M. Blennow and A. Yu. Smirnov, Adv. High Energy Phys. 2013, 972485 (2013).

[28] P. B. Denton, H. Minakata, and S. J. Parke, J. High Energy Phys. 06 (2016) 051.

[29] A. Ioannisian and S. Pokorski, arXiv:1801.10488.

[30] J. Bernabeu and A. Segarra, arXiv:1807.11879.

[31] C. Jarlskog, Z. Phys. C 29, 491 (1985).

[32] P. F. Harrison and W. G. Scott, Phys. Lett. B 476, 349 (2000).

[33] J. Bernabeu, G. C. Branco, and M. Gronau, Phys. Lett. 169B, 243 (1986).

[34] M. C. Banuls, G. Barenboim, and J. Bernabeu, Phys. Lett. B 513, 391 (2001).

[35] I. Mocioiu and R. Shrock, Phys. Rev. D 62, 053017 (2000).

[36] V. De Romeri, E. Fernandez-Martinez, and M. Sorel, J. High Energy Phys. 09 (2016) 030. 